Article

\title{
Effect of Nanoencapsulated Vitamin B1 Derivative on Inhibition of Both Mycelial Growth and Spore Germination of Fusarium oxysporum f. sp. raphani
}

\author{
Jeong Sub Cho ${ }^{1}$, Yong Chang Seo ${ }^{2}$, Tae Bin Yim ${ }^{1}$ and Hyeon Yong Lee ${ }^{3, *}$ \\ 1 DooSan EcoBizNet, Chuncheon 200-161, Korea; E-Mails: jschos@doosan.com (J.S.C.); \\ taebini@doosan.com (T.B.Y.) \\ 2 Department of Medical Biomaterials Engineering, Kangwon National University, \\ Chuncheon 200-701, Korea; E-Mail: yongchang2da@kangwon.ac.kr \\ 3 Department of Teaics, Seowon University, Cheongju, Chungbuk 361-742, Korea \\ * Author to whom correspondence should be addressed; E-Mail: hyeonl@seowon.ac.kr; \\ Tel./Fax: +82-43-299-8471.
}

Received: 20 December 2012; in revised form: 23 January 2013 / Accepted: 29 January 2013 / Published: 21 February 2013

\begin{abstract}
Nanoencapsulation of thiamine dilauryl sulfate (TDS), a vitamin B1 derivative, was proved to effectively inhibit the spore germination of Fusarium oxysporum f. sp. raphani ( $F$. oxysporum), as well as mycelial growth. The average diameter of nanoparticles was measured as $136 \mathrm{~nm}$ by being encapsulated with an edible encapsulant, lecithin, whose encapsulation efficiency was about $55 \%$ in containing 200 ppm of TDS concentration: the 100 ppm TDS nanoparticle solution showed a mycelial growth inhibition rate of 59\%. These results were about similar or even better than the cases of treating $100 \mathrm{ppm}$ of dazomet, a positive antifungal control (64\%). Moreover, kinetic analysis of inhibiting spore germination were estimated as $6.6 \%$ reduction of spore germination rates after $24 \mathrm{~h}$ treatment, which were $3.3 \%$ similar to the case of treating $100 \mathrm{ppm}$ of a positive control (dazomet) for the same treatment time. It was also found that TDS itself could work as an antifungal agent by inhibiting both mycelial growth and spore germination, even though its efficacy was lower than those of nanoparticles. Nanoparticles especially played a more efficient role in limiting the spore germination, due to their easy penetration into hard cell membranes and long resident time on the surface of the spore shell walls. In this work, it was first demonstrated that the nanoparticle of TDS not a harmful chemical can control the growth of $F$. oxysporum by using a lower dosage than commercial herbicides, as well as
\end{abstract}


the inhibiting mechanism of the TDS. However, field trials of the TDS nanoparticles encapsulated with lecithin should be further studied to be effectively used for field applications.

Keywords: thiamine dilauryl sulfate; nanoencapsulation; lecithin; spore germination; mycelial growth; Fusarium oxysporum f. sp. raphani

\section{Introduction}

White radish (Raphanus sativus) is an annual or biennial plant of the dicotyledonous papaverales crucifer family and is grown to get the large, fleshy roots. The edible part of the root has various shapes, including round, rectangular and long cylindrical, depending on the variant. It has existed widely in the temperate regions of China, South Korea, Japan and Europe [1]. One of the vegetables representing South Korea, white radish can now be grown throughout the year, thanks to the cultivation of new varieties with low thermosensitivity to low temperature and to the new subdivision of cropping patterns. The diseases and physiological disorders of white radish, however, are gradually increasing. Nineteen types of diseases in white radish have been reported to date, including Fusarium wilt and anthracnose [2].

One of the diseases of white radish, Fusarium wilt, first occurred in California, USA, and has spread all over the U.S. [3,4]. In South Korea, it was first reported in a white radish plantation in the suburbs of Chuncheon, and its incidence is gradually increasing, due to repeated cultivations $[5,6]$. Fusarium oxysporum, which causes Fusarium wilt in white radish, is a soil-borne Fungi imperfecti. It forms chlamydospores and exists in a dormant state in soil for several years, even with no host plant. Later, when the soil changes to an environment in which diseases occur, the chlamydospores germinate, enter the roots of the host plants and cause Fusarium wilt [7]. Various measures are being taken to control Fusarium wilt, such as crop rotation, soil fumigation, seed disinfection and prohibition of the overuse of nitrogen fertilizer, but these measures are not economical and have no obvious control effects. There is also no registered disinfectant for Fusarium wilt, making it difficult to control it [8]. Moreover, as most pesticides have the effect of controlling mycelial growth or killing plant disease pathogens, an efficient, reliable method for controlling spore germination is required to prevent Fusarium wilt, which starts with the germination of chlamydospores before the formation of mycelia.

Meanwhile, to prevent the spread of the aforementioned plant disease, many countries around the world, including South Korea, have recognized the need for eco-friendly biotic pesticides and are exerting much effort to develop related technologies and products. Most biotic pesticides are microbial pesticides, which are harmless to people, livestock and crops, while effectively controlling the target diseases and pests. Advanced countries have discovered beneficial microorganisms appropriate in their environments and have been utilizing these for many years. Global chemical and pesticide manufacturers have been focusing on the bioengineering projects of late and have been strengthening their strategy to advance to the biotic pesticide market. Particularly in South Korea, which has a relatively small land area and limited natural resources, there is an urgent need to maximize the 
national industrial competitiveness through the development of biotic pesticides that can actively respond to the global liberalization trend.

For this reason, the vitamin B1 derivative, thiamine dilauryl sulfate (TDS), which has been reported to have antifungal activity [9], was used in this study as a biotic pesticide. To efficiently enhance its effect of inhibiting the mycelial growth of plant disease pathogens, as well as its effect of controlling Fusarium wilt, which begins with the germination of chlamydospores, TDS was nanoencapsulated. This nanoencapsulation technology entraps the active components in the small size of $10^{-9} \mathrm{~m}$, not only to lengthen their duration, but also to adjust their activation at the desired place. Thus, its use is gradually increasing [10,11]. In this study, the possibility of using TDS as a pesticide for inhibiting spore germination through the nanoencapsulation technology, to efficiently control the spore germination of $F$. oxysporum, which causes Fusarium wilt, was explored.

\section{Results and Discussion}

\subsection{Production of TDS Nanoparticles and Identification of Their Characteristics}

Figure 1A shows the TEM photograph of the produced TDS nanoparticles that were $200 \mathrm{~nm}$ in size or smaller. It is shown that the nanoparticles were spherical and that they had a uniform size of around $120 \mathrm{~nm}$. Their dispersibility in the solution phase was also excellent. Figure 1B shows the DLS measurement of the TDS nanoparticle solution. The mean particle size distribution of the TDS nanoparticle solution was $136 \mathrm{~nm}$, which shows, along with the TEM photograph, that $150 \mathrm{~nm}$ or smaller nanoparticles were entrapped. This result suggests that entrapping TDS solution with edible lecithin makes it safe for use in food and can be directly applied to the manufacturing of spore germination inhibition pesticide. Furthermore, entrapping TDS solution in the nano size of $150 \mathrm{~nm}$ or smaller increases the surface area acting on the spores of $F$. oxysporum, as well as the spore penetration capability of TDS, thus generating a control effect greater than that of general pesticides acting on mycelia [9].

Figure 1. Characterization of the thiamine dilauryl sulfate (TDS) nanoparticle solution. (A) Photograph of the TDS nanoparticle solution from transmission electron microphotography (TEM); (B) Distribution of the TDS nanoparticle solution via dynamic light scattering (DLS).

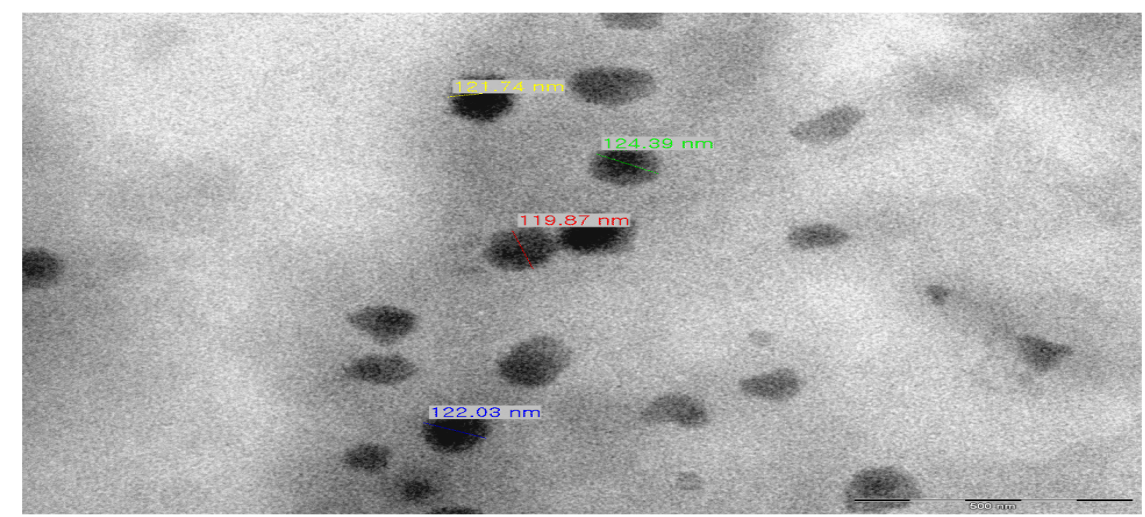

(A) 
Figure 1. Cont.

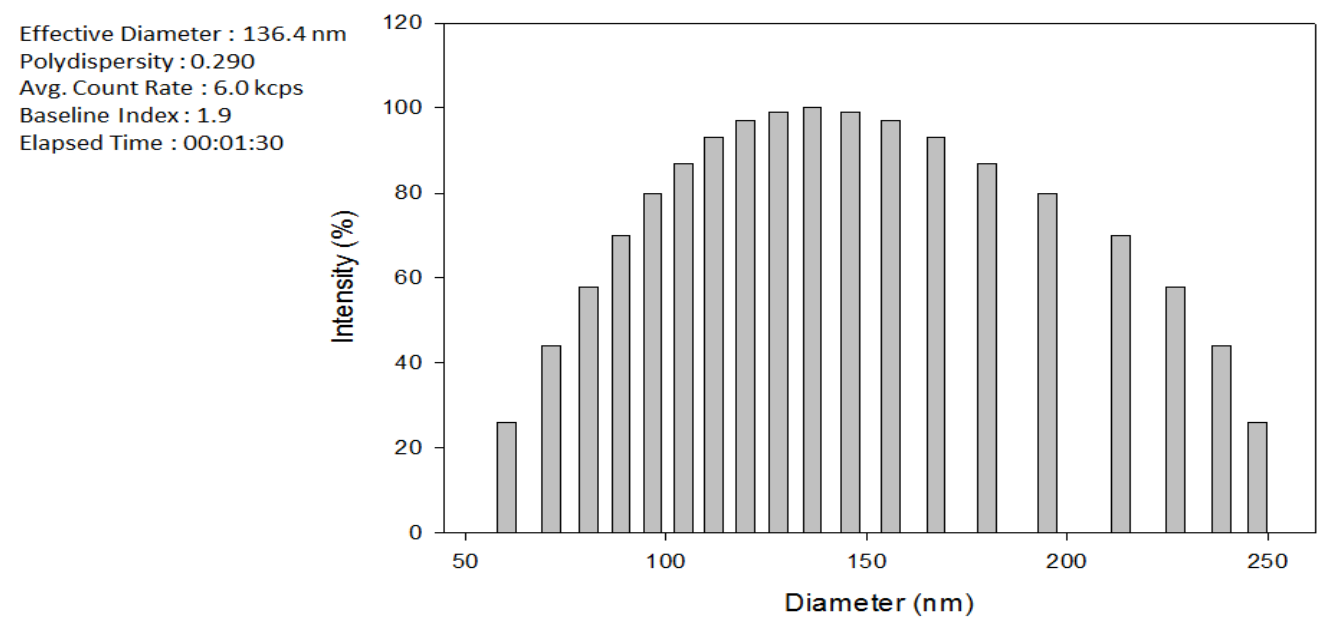

(B)

\subsection{Encapsulation Efficiency of the TDS Nanoparticle}

To determine the encapsulation efficiency of TDS in the nanoparticles, TDS concentration in the solution was measured after dissolving the lecithin nanoparticles by HPLC analysis. Figure 2A was the TDS standard (100 ppm), and Figure 2B was the peak diagram of TDS buffer solution after being dissolved by PBS buffer by containing $c a$. $55 \mathrm{ppm}$ of TDS. This result represented that the encapsulation efficiency of TDS nanoparticle with lecithin was about 55\%, whose yield was relatively highly efficiency. This result was similar to the encapsulation efficiency of other water-soluble active substances in the ranges of $40 \%-60 \%$ [12]. Based on these results, it may be concluded that lecithin is a suitable carrier for the encapsulation of TDS solution.

Figure 2. HPLC chromatograms of the TDS nanoparticle. (A) TDS standard, 100 ppm; (B) TDS after dissolving the nanoparticles.
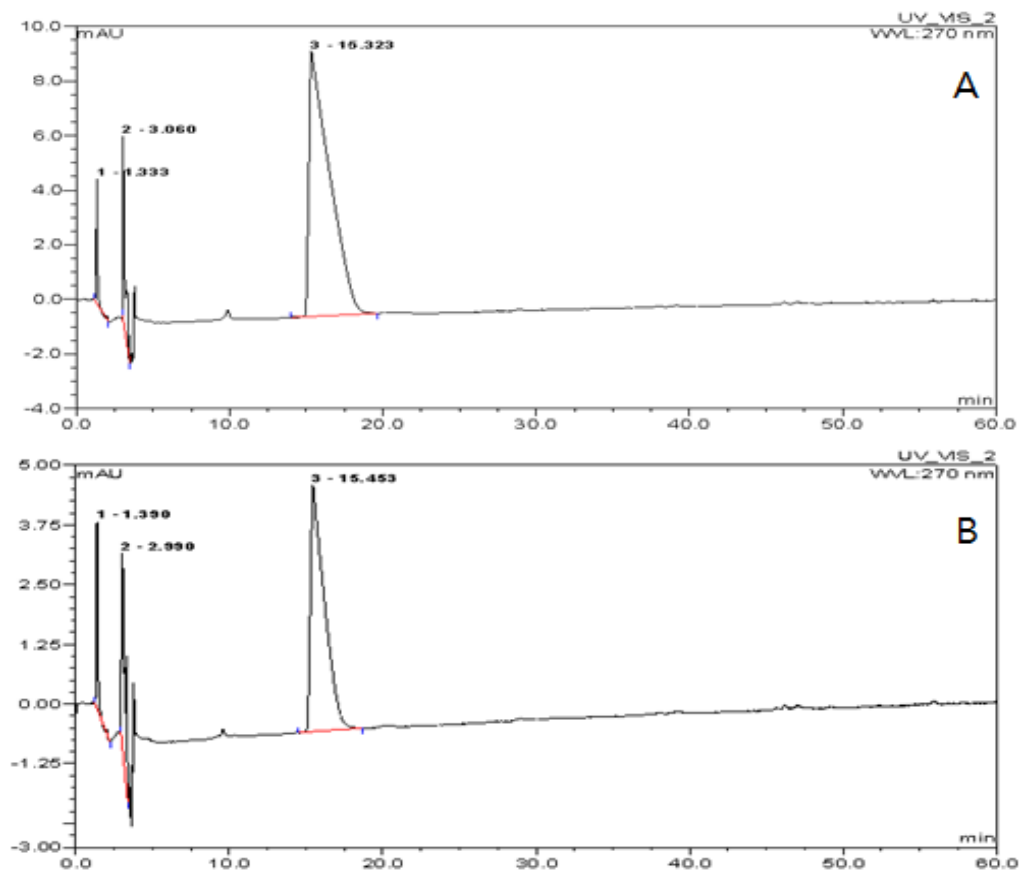


\subsection{Activation of the Inhibition of Fusarium oxysporum Mycelia Growth by the TDS}

\section{Nanoparticle Solution}

The observation of the inhibition of the mycelial growth of $F$. oxysporum by the TDS nanoparticle solution revealed that the mycelial growth inhibition effect appeared in every experimental group as opposed to the control group and that the growth inhibition effect increased along with the TDS concentration. The experimental group that showed the highest mycelial growth inhibition activity was that where $100 \mathrm{ppm}$ TDS nanoparticle solution was used, whose efficacy was similar or slightly lower than the results in adding $100 \mathrm{ppm}$ of dazomet, a positive control (Figure 3). Besides the data in Figure 3 that compared the inhibition efficacy at the end point of the experiments, kinetic analysis of inhibiting action of the samples were compared in Figure 4, according to the treatment time, whose result can better represent the action of the samples on the mycelial growth and spore germination. A mycelial growth inhibition rate of $38 \%$ was shown in adding $100 \mathrm{ppm}$ of TDS itself, while the highest mycelial growth inhibition rate of 59\% was shown in adding the same concentration of TDS nanoparticles, even though $64 \%$ of the highest inhibition was observed in adding a commercial herbicide, dazomet. For the case of adding lecithin (an encapsulant) itself, (G) in Figure 4, the mycelia growth was also almost similar to the case of no treatment (A). This result implied that the lecithin did not show a positive or negative effect on the mycelia growth for this experiment. These results suggest that using TDS nanoparticle solution can dramatically increase the surface areas acting on the mycelia of F. oxysporum, as well as the persistence of TDS in the mycelia, as opposed to using general TDS solution $[13,14]$. Moreover, whereas the water-dispersible powder of metalaxyl-copper oxychloride, a known chemical sterilizer, showed a 50\% inhibition rate, the $100 \mathrm{ppm}$ TDS nanoparticle solution showed a higher mycelial growth inhibition rate of $60 \%$ [15].

Figure 3. Comparison of the antifungal activities in the $20 \mathrm{~mL}$ TDS solution and $20 \mathrm{~mL}$ TDS nanoparticle solution for two different concentrations. (A) Negative control (no treatment); (B) positive control (dazomet), 100 ppm; (C) TDS solution, 50 ppm; (D) TDS solution, 100 ppm; (E) TDS nanoparticle solution, 50 ppm; (F) TDS nanoparticle solution, 100 ppm; (G) empty lecithin vesicles.

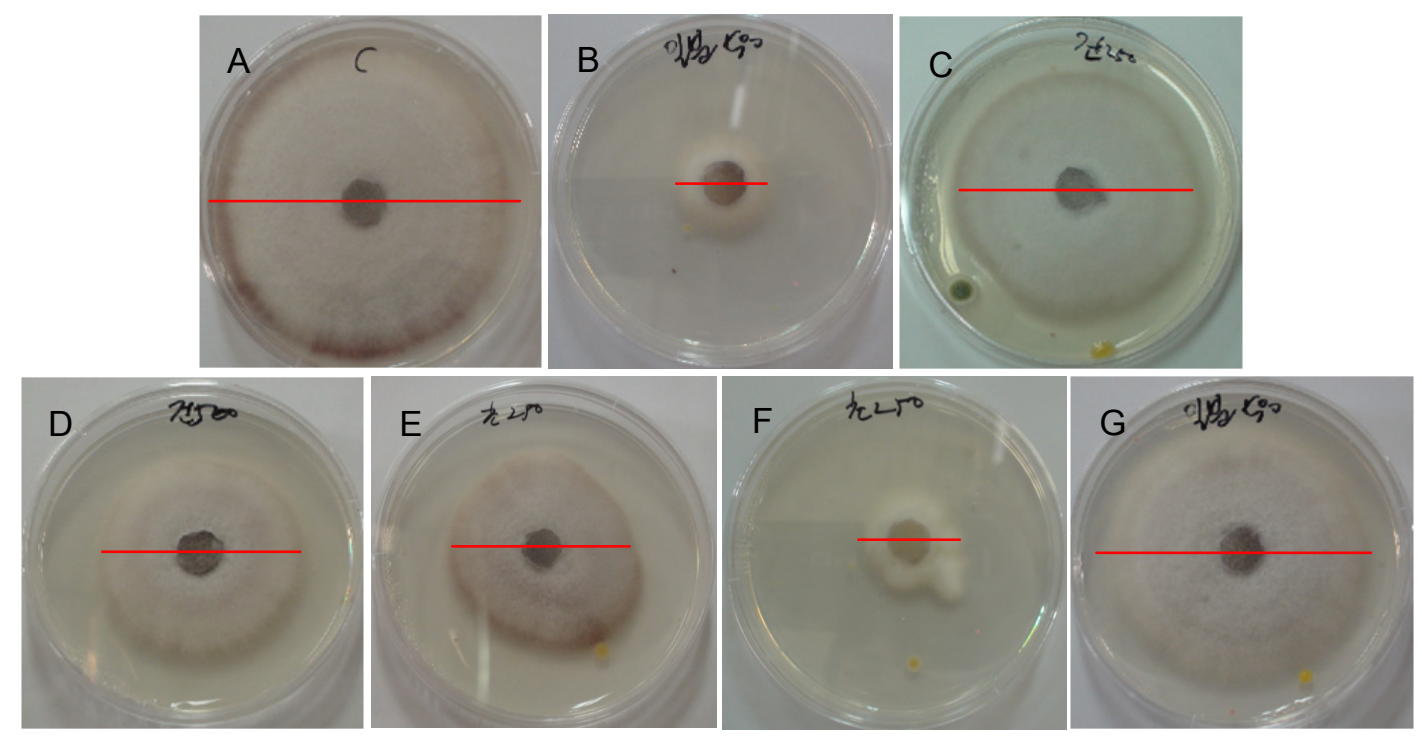


Figure 4. Growth of Fusarium oxysporum f. sp. raphani when different concentrations of TDS solution and TDS nanoparticle solution were added to it. (A) Negative control (no treatment); (B) positive control (dazomet), 100 ppm; (C) TDS solution, 50 ppm; (D) TDS solution, 100 ppm; (E) TDS nanoparticle solution, 50 ppm; (F) TDS nanoparticle solution, 100 ppm; (G) empty lecithin vesicles.

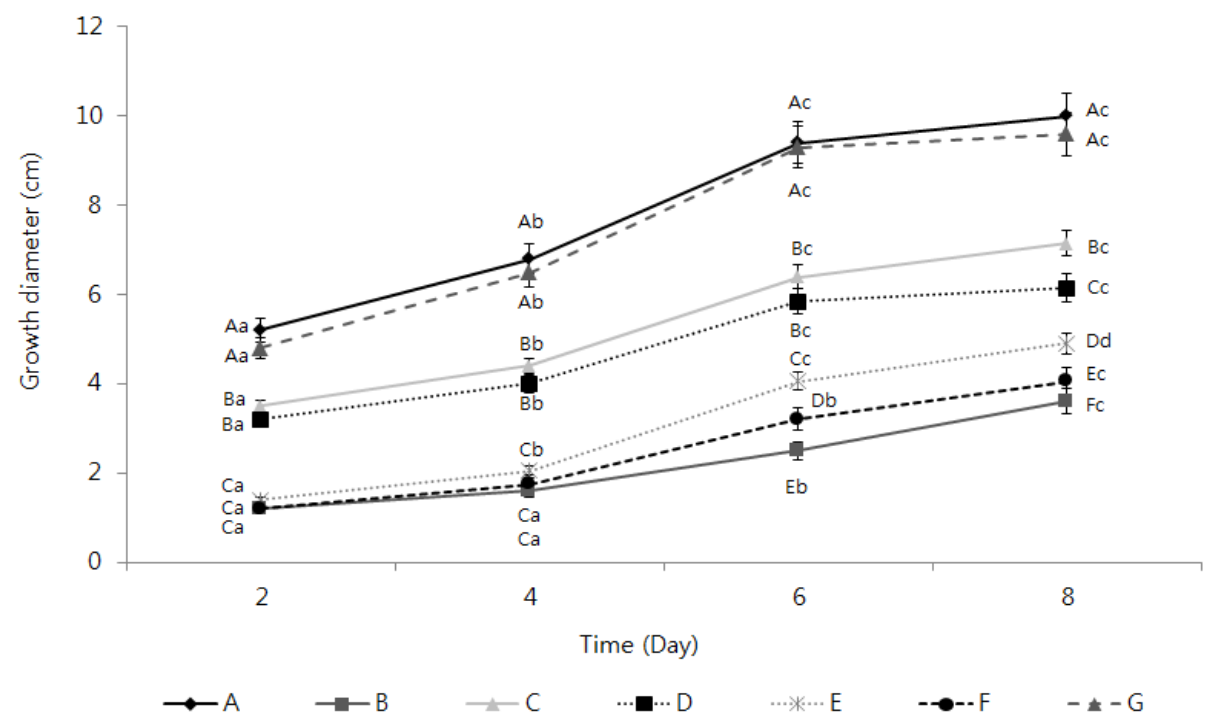

\subsection{Measurement of the Spore Germination Inhibition Effect of the TDS Nanoparticle Solution}

To examine the spore germination inhibition effect of $100 \mathrm{ppm}$ TDS nanoparticle solution, the spore germination was observed under an optical microscope at 0,12 and $24 \mathrm{~h}$ (Figure 5). For the case of negative control (no treatment), spores were propagated to $1.29 \times 10^{3}$ conidia/mL and fully germinated to the mycelia within $24 \mathrm{~h}$ after the inoculation, while the number of the spores was slowly increased only to $6.5 \times 10^{1}$ conidia/mL and no visible growth of mycelia after $24 \mathrm{~h}$ in adding $100 \mathrm{ppm}$ of TDS nanoparticles. This result implies that the TDS nanoparticle effectively inhibited the germination of chlamydospores and resulted in decreasing the mycelia growth.

To examine this spore germination inhibition effect in greater detail, the spore germination rate was measured by comparing with TDS itself and a commercial herbicide (Figure 6). The 100 ppm TDS solution showed the spore germination rates of $56.1 \%$ at $12 \mathrm{~h}$ and $28.6 \%$ at $24 \mathrm{~h}$. In comparison, the $100 \mathrm{ppm}$ TDS nanoparticle solution showed the spore germination rates of $22.3 \%$ at $12 \mathrm{~h}$ and greatly decreased down to $6.6 \%$ after $24 \mathrm{~h}$, where the inhibition of the germination was very similar to the case of adding the dazomet (3.7\% germination), even though for the first $12 \mathrm{~h}$ there was about a 15\%-20\% difference between the cases of TDS nanoparticle and dazomet. This result would suggest that TDS encapsulated nanoparticles required the time to start inhibiting the germination, compared to a herbicide, even though the nanoparticles can more effectively control the germination than TDS itself, possibly due to large surface areas and small sizes, enough to penetrate into the cell membrane and work in the cytosols [13]. This hypothesis can be proven by electron microscope observation of breaking the germs, as well as kinetic penetration of TDS nanoparticles into the germs, as shown in Figures 7 and 8. 
Figure 5. Spore inhibition in Fusarium oxysporum f. sp. raphani by TDS nanoparticle solution under microscope observation. (A) Negative control (no treatment); (B) TDS nanoparticle solution, $100 \mathrm{ppm}$.

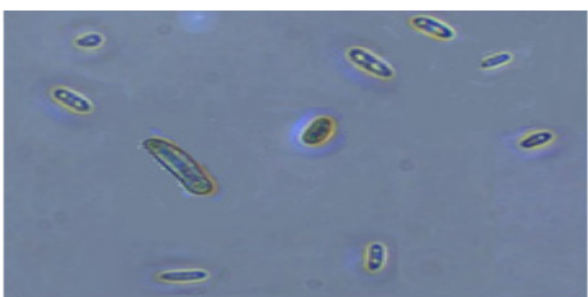

$\mathbf{A}(0 \mathrm{~h})$

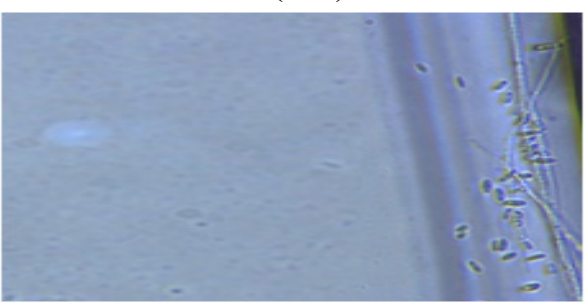

$\mathbf{A}(12 \mathrm{~h})$

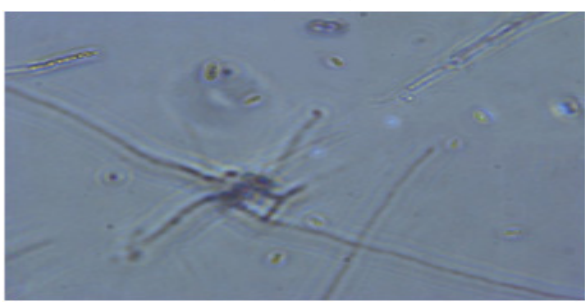

$\mathbf{A}(24 \mathrm{~h})$

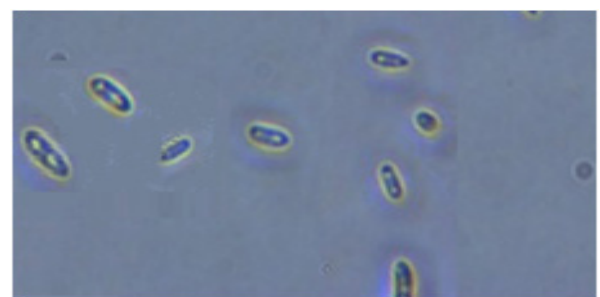

B $(0$ h)

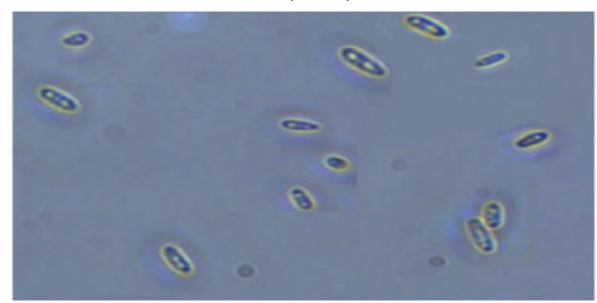

B $(12 \mathrm{~h})$

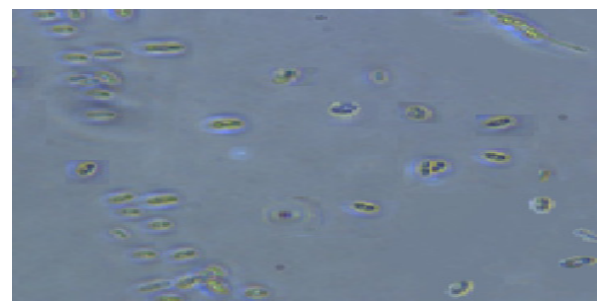

B $(24 \mathrm{~h})$

Figure 6. Effects of the TDS solution and TDS nanoparticle solution on Fusarium oxysporum f. sp. raphani spore germination. (A) TDS solution, 100 ppm; (B) TDS nanoparticle solution, $100 \mathrm{ppm}$; (C) positive control (dazomet), $100 \mathrm{ppm}$.

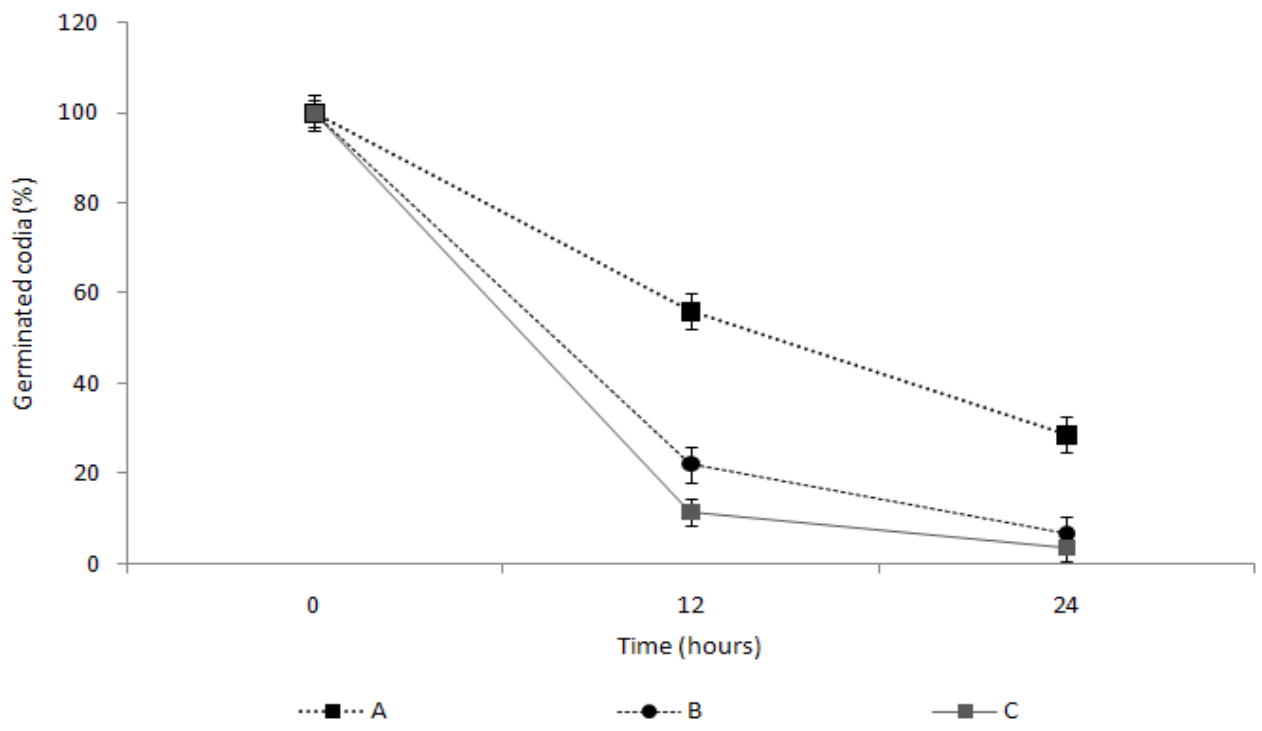




\subsection{Observation of the Spore Extinction Effect of the TDS Nanoparticle Solution}

The spores were photographed under a scanning electron microscope (SEM) to observe the spore extinction mechanism of the TDS nanoparticle solution. Figure 7A shows the mycelia in the control group (without treating TDS nanoparticles), around which spores were well formed and even seemed to start germination. In comparison, Figure 7B shows that at $0 \mathrm{~h}$, all of the spores in the disc looked like normal, but at $24 \mathrm{~h}$, most of the spores were broken and started to be digested when adding 100 ppm of TDS nanoparticles.

Figure 7. SEM images of the spores in Fusarium oxysporum f. sp. raphani after TDS nanoparticle solution treatment. (A) Negative control (no treatment) after $24 \mathrm{~h}$ incubation; (B) TDS nanoparticle solution, $100 \mathrm{ppm}$.

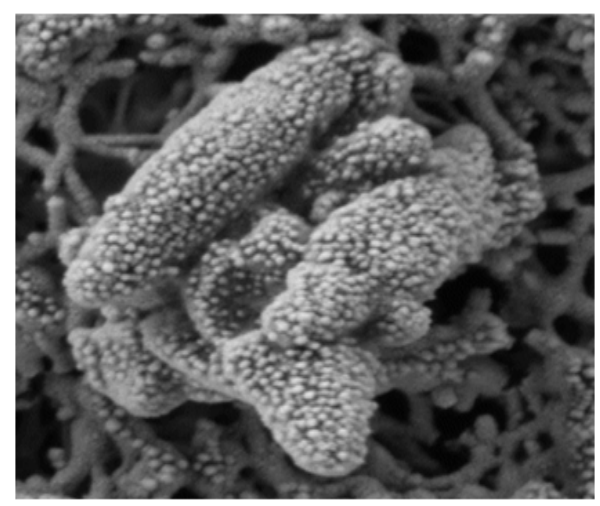

A

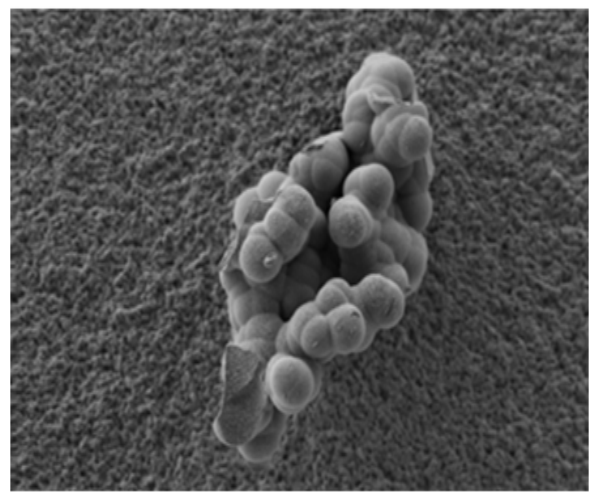

B $(0 \mathrm{~h})$

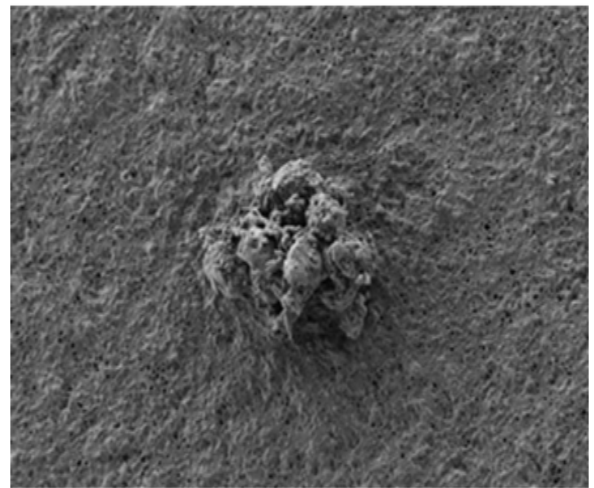

B $(24 \mathrm{~h})$

To understand the inhibition mechanism of the TDS nanoparticle, the penetration of TDS nanoparticle into the germs was observed by confocal microscope, according to the treatment time in Figure 8. From the results, it was confirmed that TDS nanoparticles could penetrate into the spore membrane within $12 \mathrm{~h}$ and started to inhibit normal functions of the many kinds of molecules in the cytosol. After $12 \mathrm{~h}$, FITC fluorescence started to be observed inside the spores and was evenly distributed throughout the spores after $6 \mathrm{~h}$ and completely covered the disc by breaking all of the germs. However, for the case of adding $100 \mathrm{ppm}$ of TDS itself, the FITC labeled TDS was slowly passed through the cell membrane after $6 \mathrm{~h}$, and the penetration was not very developed, even after $12 \mathrm{~h}$ treatment. This real-time confocal observation result first reported that the TDS nanoparticles can penetrate into the germs that are known to be very resistant and can work on inhibiting cell functions. 
It was suggested that cellular uptake of nanoparticles is more effective than uptake of micro-sized particles, owing to sustained-release properties, sub-cellular size and the target ability to a cell [16]. For a further detailed explanation of this observation, the effect of nanoparticle size on inhibiting the germination of the spores would be carried out, since most of the nanoparticle size was in the range of $150-200 \mathrm{~nm}$ in this experiment. A smaller particle size would be more effective in penetrating into the cell membrane, even though it would not be easy to make smaller sized nanoparticles. The tolerance of fungi towards chemicals may be due to changes in biochemistry of fungal cell walls that inhibit the entry of pesticides inside cells to a greater or lesser extent and, thereby, not reaching the site of action. Such changes may result in the decrease of the permeability of cell membrane and pesticidal detoxification, even before the site of action [17]. Conversion of a chemical in an active form may also be responsible for the detoxification mechanism [18].

Figure 8. Confocal microscope images of the spores in Fusarium oxysporum f. sp. raphani in adding TDS nanoparticle solution, according to the treatment time. (A) TDS nanoparticle solution, 100 ppm; (B) TDS solution, 100 ppm.

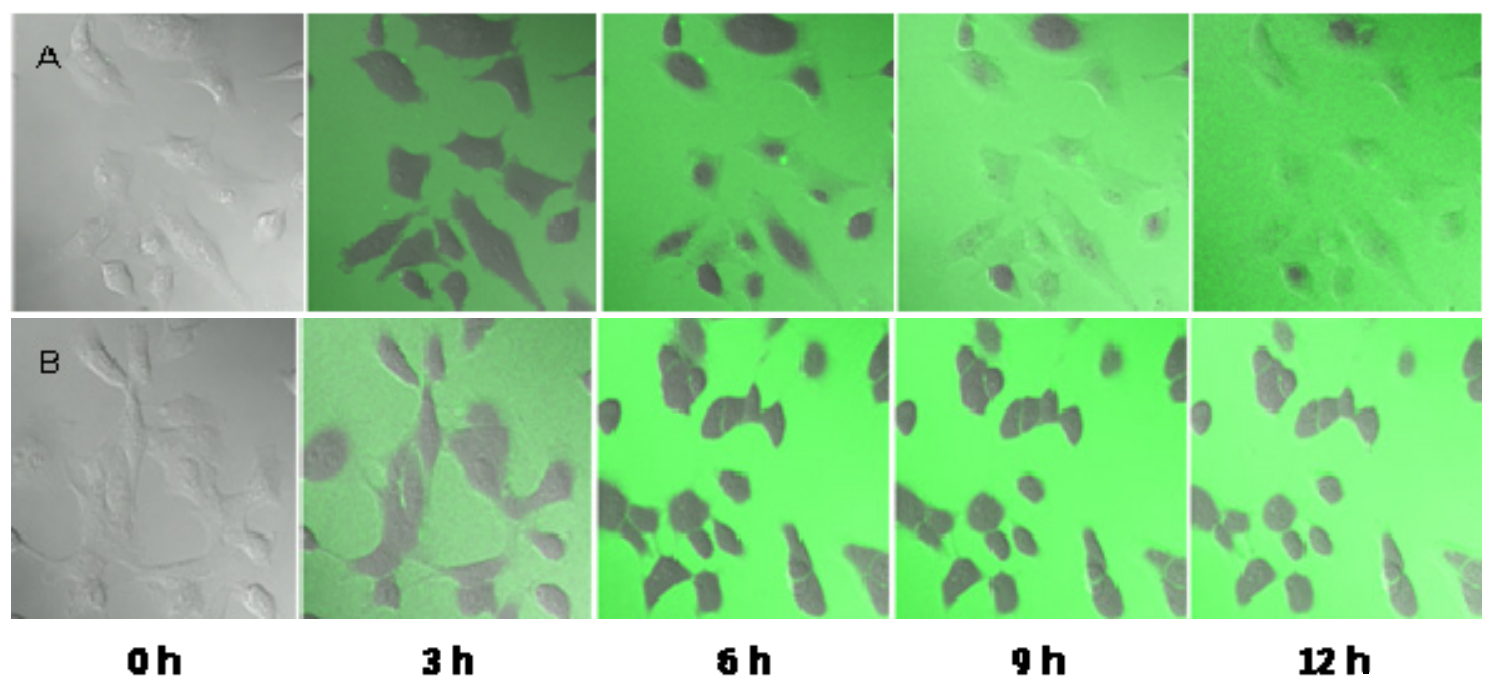

\section{Experimental Section}

\subsection{Cultivation of White Radish}

Songbaek radish seeds were used for the experiment to establish the disease resistance test method. Horticultural bedsoil No. 5 (Bunongsa, Korea) was input into $8 \times 16$ linked pots $(20 \mathrm{~mL}$ soil per pot, Bumnong, Korea), and the radish seeds were grown in the pots before they were cultivated in a greenhouse $\left(25 \pm 5^{\circ} \mathrm{C}\right)$ for 14 days.

\subsection{Fusarium oxysporum $f$. sp. raphani Spores}

Fusarium oxysporum f. sp. raphani (KACC 40146, Korea) was used to investigate the spore germination inhibition activity. For the culture medium, potato dextrose agar (PDA, Sigma-aldrich, St. Louis, MO, USA) was used. The strains were cultivated at $25{ }^{\circ} \mathrm{C}$ for seven days, and the plectenchyma were removed from the colonies and were inoculated to malt extract broth 
(Becton, Dickinson and Co., Seoul, Korea). They were then shake-cultured at $150 \mathrm{rpm}$ at $25^{\circ} \mathrm{C}$ in a dark state for seven days. The cultivated strains were filtered through four layers of gauze to remove the hyphae, and the spore concentration was measured with a hemocytometer under an optical microscope (Zeiss Axioskop Microscope, Heidelberg, Germany). The samples were diluted with sterile water to the spore concentration of $1.0 \times 10^{-7}$ conidia $/ \mathrm{mL}$.

\subsection{Production of TDS Nanoparticle Solution}

To produce the TDS solution that was used in this experiment, $100 \mathrm{~g}$ TDS powder was put in $1 \mathrm{~L}$ of $60 \%$ alcohol and was stirred for about $10 \mathrm{~min}$, until it was completely dissolved. One milliliter of this TDS solution was added to $499 \mathrm{~mL}$ distilled water, and the resulting mixture was stirred for $60 \mathrm{~min}$ to produce $200 \mathrm{ppm}$ TDS solution. To improve the safety of the crops, lecithin, which is edible, was used for the nanoencapsulation of the TDS solution. For lecithin, L- $\alpha$-phosphatidylcholine (Sigma-aldrich, St. Louis, MO, USA) was used. It was melted in chloroform (Sigma-aldrich, St. Louis, MO, USA) and was put in a round bottom flask. All the chloroform was evaporated in a decompressed state to form lecithin multilayers. The TDS solution was added to the round bottom flask in which multiple lecithin layers were formed after complete drying and was homogenized at room temperature for one hour to produce TDS nanoparticle solution [19]. To collect $200 \mathrm{~nm}$ or smaller particles, it was filtered with a $0.2 \mu \mathrm{m}$ syringe filter.

\subsection{Observation of the TDS Nanoparticles}

To check the particle shape of the produced TDS nanoparticles, the TDS nanoparticles were negative-stained with a phosphotungstic acid solution and were fixed with formvar/carbon. They were then thinly spread on a grid and dried. Then the nanoparticles were photographed under a transmission electron microscope (EF-TEM, LEO 912AB Omega, Carl Zeiss, Oberkochen, Germany) at $120 \mathrm{kV}$ to capture their sizes and shapes [20]. To measure the uniformity and size distribution of the produced TDS nanoparticle solution, the size distribution of the TDS nanoparticles was measured via dynamic light scattering (DLS, Brookhaven Instruments Co., New York, NY, USA).

\subsection{Measurement of Encapsulation Efficiency of the TDS Nanoparticle}

The oversized nanoparticles in the solution were removed by gel-permeation chromatography using Sephadex G-100 columns $(1.6 \mathrm{~cm} \times 40 \mathrm{~cm}$; bead size 40-120 $\mu \mathrm{m})$ purchased from GE Healthcare (Uppsala, Sweden). The collected TDS nanoparticle fraction was centrifuged for $30 \mathrm{~min}$ at 16,770× $g$, and the precipitate was dissolved by adding $25 \mathrm{~mL}$ acetone (Sigma, St. Louis, MO, USA). After $30 \mathrm{~min}$ of stirring, $250 \mathrm{mg}$ L-cysteine (Sigma, St. Louis, MO, USA) was added. The sample was sonicated at $60 \mathrm{kHz}$ for $30 \mathrm{~min}$ and filtered through a $0.2 \mu \mathrm{m}$ syringe filter [21]. The content of TDS in the filtrate was then determined by HPLC (M600E, M7725i/Waters, 996PDA, Waters, Milford, MA, USA). For HPLC analysis, a C18 column $(250 \times 4.6 \mathrm{~mm}$, Waters, Milford, MA, USA) was used, where the mobile phase was water and acetonitrile and the gradient ratio was water:acetonitrile $=80: 20$ $\sim 20: 80$ at $1.0 \mathrm{~mL} / \mathrm{min}$ of flow rate and $270 \mathrm{~nm}$ absorbance. The injection volume was $25 \mu \mathrm{L}$. 


\subsection{Measurement of the F. oxysporum Mycelia Inhibition Effect of the TDS Nanoparticle Solution}

The antifungal activity was measured to examine the $F$. oxysporum mycelia inhibition activity of the TDS nanoparticle solution. Strains of Fusarium oxysporum f. sp. raphani (KACC 40146, Korea) received from the National Institute of Agricultural Science and Technology were used for this experiment. Potato dextrose agar (PDA, Sigma-aldrich, St. Louis, MO, USA) was used as the culture medium, and the samples were cultivated at $20{ }^{\circ} \mathrm{C}$ in a dark state. For the measurement of the antifungal activity, a modified version of the method developed in [22] was used. For the experimental groups, general TDS solution and TDS nanoparticle solution were diluted to 50 and $100 \mathrm{ppm}$, respectively, and $20 \mathrm{~mL}$ volumes were used. A commercial herbicide, dazomet (3-5-dimethyltetrahydro-1-3-5-2 $\mathrm{H}$ thiadiazine 2 thione), was also used as a positive control. Each sample was added when the PDA culture media were produced. $F$. oxysporum strains were cultivated at $20{ }^{\circ} \mathrm{C}$ in a dark state for five days. A $0.5 \mathrm{~cm}$ colony was inoculated at the center of each of the media containing TDS solution and TDS nanoparticle solution, and the growth of the colonies was observed. To examine the growth inhibition rate in detail, the colony diameter was measured by date, and the mycelial growth inhibition rate was measured using the following formula. The experiment was conducted three times for each treatment group [9].

mycelial growth inhibition rate $(\%)=(1-$ diameter of mycelia in the TDS-treated medium/diameter of mycelia in the no-treatment medium) $\times 100$

\subsection{Measurement of the Spore Germination Inhibition Effect of the TDS Nanoparticle Solution}

To examine the spore germination inhibition effect of the TDS nanoparticle solution, a PDA medium was produced with no TDS nanoparticle solution. For the experimental groups, TDS solution and TDS nanoparticle solution were added to the final concentration of $100 \mathrm{ppm}$ during the production of the PDA medium, as well as the same concentration of 3-5-dimethyltetrahydro-1-3-5-2 H thiadiazine 2 thione (dazomet) as a positive control. For the control group and for all the experimental groups, spores with $1.0 \times 10^{3}$ conidia/mL concentration were inoculated and cultivated at $25{ }^{\circ} \mathrm{C}$ in a dark state. After some time, the media on which the spores were cultivated were cut into $1 \times 1 \mathrm{~cm}$ pieces and were observed under an optical microscope (Zeiss Axioskop Microscope, Oberkochen, Germany). To examine the spore growth inhibition rate, spores with $1.0 \times 10^{3}$ conidia $/ \mathrm{mL}$ concentration were inoculated to the media of the control and experimental groups. Then, they were cultivated at $25{ }^{\circ} \mathrm{C}$ in a dark state. The spore concentrations in the media cut into $2 \times 2 \mathrm{~cm}$ pieces were measured over time. Furthermore, to examine the spore germination rate in detail, it was measured with the following formula using the spore concentration measured by hour. Three experiments were conducted for each treatment group.

spore germination rate $(\%)=($ spore concentration in the sample-treated medium/spore concentration in the no-treatment medium) $\times 100$

\subsection{Observation of the Spore Extinction Effect of the TDS Nanoparticle Solution}

To examine the mechanism of the TDS nanoparticle solution's induction of spore extinction, the spores cultivated in the medium to which TDS nanoparticle solution was added during the 
measurement of the spore germination inhibition effect were observed. A scanning electron microscope was used to measure the surface. For the pretreatment, fresh samples were fixed in $2.5 \%$ glutaraldehyde solution at room temperature for $3 \mathrm{~h}$, were washed with a $0.1 \mathrm{M}$ phosphate buffer and were dehydrated with alcohol series. After dehydration, the samples were dried with a $\mathrm{CO}_{2}$ critical point drier (CPD2, Pelko, Melbourne, FL, USA), and the sample surfaces were gold-coated with a goldcoater (SC7640 sputter coater, Pocaron, Cambridge, UK) for $2 \mathrm{~min}$. They were then observed with an electron microscope (LEO-435VP, SEM, North Billerica, MA, USA). Also, to image the penetration of TDS nanoparticles into the spore, a confocal laser scanning microscope (LSM510 META NLO, Carl Zeiss, Jena, Germany) was employed as follows: spores were prepared in the confocal dish; $200 \mu \mathrm{L}$ of nanoparticles containing a fluorescein isothiocyanate (FITC) labeled TDS solution were then applied onto the spore and observed every $12 \mathrm{~h}$ until the germs were completely broken. The cross-section was imaged at $543 \mathrm{~nm}$ using a confocal laser scanning microscope [23].

\subsection{Statistical Processing}

The statistics of the experimental values were verified through paired $t$-test with the SPSS application. All the experimental values were expressed as mean \pm standard error.

\section{Conclusions}

In this study, the vitamin B1 derivative thiamine dilauryl sulfate (TDS), which has been reported to have antifungal activity, was nanoencapsulated, and its effects in terms of the inhibition of the mycelial growth and spore germination of Fusarium wilt were investigated. Entrapping TDS solution with edible lecithin via nanoencapsulation makes the solution safe to use in food and allows it to be directly applied to the manufacturing of spore germination inhibition pesticide. Furthermore, entrapping TDS solution in the nano size of $150 \mathrm{~nm}$ or smaller increases the surface area acting on the spores of F. oxysporum, as well as the spore penetration capability of TDS, thus generating a control effect greater than that of general pesticides acting on mycelia [9]. This nanoencapsulation technology is used to entrap TDS, which has low stability in solution, as nano-sized particles and to increase its dispersibility in solution, thereby overcoming its low stability [24].

Using TDS nanoparticle solution increased the surface area acting on the mycelia of $F$. oxysporum, as well as easy penetration into the rigid germs [13], compared to TDS itself, to higher inhibition of mycelial growth and germination. Once TDS nanoparticles passed through the cell membranes of mycelia or spores, TDS can effectively inhibit the cell metabolisms, because being a vitamin B1 derivatives, TDS can strongly attach to the cell membranes and inhibit cell differentiation and proliferation in the cytosol [25]. According to this mechanism, the TDS nanoparticles inhibit cell division by attaching to the cell membranes of the mycelia or substantially inhibiting their proliferation by penetrating the protoplasm [26]. It showed greater mycelial growth inhibition activity than chemical pesticide, which is a water-dispersible powder of metalaxyl-copper oxychloride, a known chemical sterilizer. Entrapping the nanoparticles with edible lecithin improved the safety and decreased the residual toxicity, which is a problem of chemical pesticide, thus making it a safe biotic pesticide.

The measurement of the spore germination inhibition effect against $F$. oxysporum, which begins with the germination of chlamydospores before the formation of mycelia, showed the lowest spore 
germination rate in the TDS nanoparticle solution. The lecithin nanoparticles with hydrophobic surfaces were 100-300 $\mathrm{nm}$ in size and could easily penetrate the cells and facilitate the transportation of molecular matter, increasing the affinity to the cells [27]. Moreover, to improve TDS's capability of penetrating the cells of soluble materials, many studies are being conducted to provide TDS with high penetration power and bio-utility by manufacturing active materials entrapped by oil as W/O-type liposomes [28]. The TDS nanoparticles in this study improved TDS's persistence in the spores, its affinity and its spore penetration capability. The spore germination inhibition effect of the TDS nanoparticle solution showed a value similar to the reported spore germination inhibition effect value of hexanoic acid against Botrytis cinerea in tomato. Similar to the mechanism of acidifying the protoplasm to increase its cell membrane penetration capability, TDS was nanoencapsulated with lecithin, which has a hydrophobic surface and improved affinity to the cell membrane consisting of phosphatide, thereby improving the cell penetration capability of TDS [29]. Compared to the conventional pesticides inhibiting mycelial growth, it can control $F$. oxysporum, which begins with the germination of chlamydospores before the formation of mycelia, even with a low concentration. Furthermore, TDS nanoparticles can be used as an economic pesticide for the efficient control of spore germination.

\section{Acknowledgements}

This research was financially supported by the Ministry of Knowledge Economy (MKE) and Korea Institute for Advancement of Technology (KIAT) through the Research and Development for Regional Industry (2010-2013).

\section{References}

1. Ku, K.H.; Lee, K.A.; Kim, Y.L.; Lee, Y.W. Quality characteristics of hat-air dried radish (Raphanus sativus L.) leaves. J. Korea Soc. Food Sci. Nutr. 2006, 35, 780-785.

2. Baik, S.Y.; Kim, J.C.; Jang, K.S.; Choi, Y.H.; Choi, G.J. Development of effective screening method and evaluation of radish cultivars for resistance to Fusarium oxysporum f. sp. raphani. Res. Plant Dis. 2010, 16, 148-152.

3. Pound, G.S. Red prince is new radish. Annu. Rep. Agric. Exp. Stn. 1959, 538, 93-97.

4. Pound, G.S.; Fowler, D.L. Fusarium wilt of radish in Wisconsin. Phytopathology 1953, 43, 277-280.

5. Du Toit, L.J.; Inglis, D.A.; Pelter, G.Q. Fusarium proliferatum pathogenic on onion bulbs in Washington. Plant Dis. 2003, 87, 750-756.

6. Moon, Y.G.; Kim, W.G.; Cho, W.D.; Sung, J.M. Occurrence of Fusarium wilt on cruciferous vegetable crops and pathogenic differentiation of the causal fungus. Res. Plant Dis. 2001, 7, 93-101.

7. Van Peer, R.; Xu, T.; Rattink, H.; Schippers, B. Biological control of carnation wilt caused by Fusarium oxysporum f. sp. dianthi in hydroponic system. ISOSC Proc. 1988, 361-373.

8. Alabouvette, C.; Schippers, B.; Lemanceau, P.; Bakker, P.A.H.M. Biological Control of Fusarium wilts. Plant-Microbe Interactions and Biological Control; Marcel Dekker: New York, NY, USA, 1998; pp. 15-36. 
9. Seo, Y.C.; Cho, J.S.; Jeong, H.Y.; Yim, T.B.; Cho, K.S.; Lee, T.W.; Jeong, M.H.; Lee, G.H.; Kin, S.I.; Yoon, W.B.; et al. Enhancement of antifungal activity of anthracnose in pepper by nanopaticles of thiamine dilauryl sulfate. Korean J. Med. Crop Sci. 2011, 19, 198-240.

10. Seo, D.S.; Kim, J.C.; Sohn, H.H.; Cho, W.G.; Lee, S.U.; Kim, E.Y.; Tae, G.Y.; Kim, J.D.; Lee, S.Y.; et al. Preparation and characterization of chitosan/gelatin microcapsules containing triclosan. J. Colloid Interface Sci. 2004, 273, 596-603.

11. Kim, J.C.; Lee, H.Y.; Kim, M.H.; Lee, H.J.; Kang, H.Y.; Kim, S.M. Preparation and characterization of chitosan/gelatin microcapsules containing triclosan. Colloids Surf. B 2006, 52, $52-56$.

12. Li, D.-C.; Zhong, X.-K.; Zeng, Z.-P.; Jiang, J.-G.; Li, L.; Zhao, M.-M.; Yang, X.-Q.; Chen, J.; Zhang, B.-S.; Zhao, Q.-Z.; et al. Application of targeted drug delivery system in Chinese medicine. J. Control. Release 2009, 138, 103-112.

13. Seo, Y.C.; Jeong, M.H.; Jeong, H.S.; Kim, J.S.; Zou, Y.Y.; Ahn, J.H.; Shin, I.S.; Lee, H.Y. Enhancement of antimicrobial activity of nano-encapsulated horseradish aqueous extracts against food-borne pathogens. Korean J. Med. Crop Sci.2010, 18, 389-397.

14. Seo, Y.C.; Choi, Y.W.; Lee, C.G.; Cho, J.S.; Yim, T.B.; Jeong, M.H.; Kim, S.I.; Yoon, W.B.; Lee, H.Y. Effect of solubility of thiamine dilauryl sulfate solution through the manufacture of the nano paticles on antifungal activity. Korean J. Med. Crop Sci. 2011, 19, 464-471.

15. Kim, C.H.; Yang, S.S.; Hahn, K.D. Effects of soil disinfection, fungicide application, and narrow ridge cultivation on development of ginger rhizome rot caused by Pythium myriothlum in fields. Korean J. Plant Pathol. 1998, 14, 253-259.

16. Vasir, J.K.; Reddy, M.K.; Labhasetwar, V.D. Nanosystems in drug targeting: Opportunities and challenges. Curr. Nanosci. 2005, 1, 47-64.

17. Nakanishi, T.; Oku, H. Metabolism and accumulation of pentachloronitrobenzene by phytopathogenic fungi in relation to selective toxicity. Phytopathology 1969, 59, 1761-1765.

18. Dekker, J. Acquired resistance to fungicides. Annu. Rev. Phytopathol. 1976, 14, 405-428.

19. Fallouh, N.A.; Treupel, L.R.; Fessi, H.; Devissaguet, J.; Puisieux, F. Development of a new process for the manufacture of polyisobutylcyanoacrylate nanocapsules. Int. J. Pharm. 1986, 28, 125-132.18.

20. Saxena, A.; Sachin, K.; Bohidar, H.B.; Verma, A.K. Effect of molecular weight heterogeneity on drug encapsulation efficiency of gelatin nano-particles. Colloids Surf. B 2005, 45, 42-48.

21. Ramachandran, R.; Shanmughavel, P. Preparation and characterization of biopolymeric nanoparticles used in drug delivery. Indian J. Biochem. Biophys. 2010, 47, 56-59.

22. Ko, S.W.; So, I.S.; Huh, M.R. Study on antifungal activity of Aloe arborescens M. for a potential bio-pestcide. J. Agric. Life Sci. 2009, 43, 35-44.

23. Savini, G.; Dunsmore, J.D.; Robertson, I.D. Studies on pathogenesis, tissue infection and congenital transmission in cows experimentally infected with Sarcocystis cruzi by various routes. Vet. Parasitol. 1996, 64, 319-327.

24. Han, J.G.; Kwon, M.C.; Ha, J.H.; Jeong, H.S.; Kim, Y.; Jeong, M.H.; Kim, J.C.; Lee, H.Y. Enhancement of immuno modulatory activities of Rubus coreanus miquel extracts by nano-encapsulation process. Korean J. Med. Crop Sci. 2009, 17, 54-60. 
25. Kim, S.W.; Jung, J.H.; Lamsal, K.; Kim, Y.S.; Sim, S.J.; Kim, H.S.; Chang, S.J.; Kim, J.K.; Kim, K.S.; Lee, Y.S. Control efficacy of nano-silver liquid on Oak wilt caused by Raffaelea sp. in the field. Res. Plant Dis. 2011, 17, 136-141.

26. Lee, Y.H. Use of Vitamin B1 as Agents for Controlling Plantdiseases. Korea Patent 7,011,615, 2002.

27. Rhaese, S.; Vonbriesen, H.; Rubsamen-Waigmann, H.; Kreuter, J.; Langer, K. Human serum albumin-polyethylenimine nanoparticles for gene delivery. J. Control. Release 2003, 92, 199-208.

28. Jochen, W.; Paul, T.; Julian, M. Functional materials in food nanotechnology. J. Food Sci. 2006, 71, 107-116.

29. Leyva, M.O.; Vicedo, B.; Finiti, I.; Flors, V.; Del Amo, G.; Real, M.D.; García-Agustín P.; González-Bosch, C. Preventive and post-infection control of Botrytis cinerea in tomato plants by hexanoic acid. Plant Pathol. 2008, 57, 1038-1046.

(C) 2013 by the authors; licensee MDPI, Basel, Switzerland. This article is an open access article distributed under the terms and conditions of the Creative Commons Attribution license (http://creativecommons.org/licenses/by/3.0/). 